\title{
Análise de Convergência da Renda em Santa Catarina entre 2001 e 2012: PIB per capita, Espacialidade, Renda Pessoal e Demografia
}

\author{
Convergence Analysis of Income in Santa Catarina Between 2001 na 2012: GDP per \\ capita, spatiality, personal income and demographics
}

Krisley Mendes ${ }^{1}$

Fábio Nobuo Nishimura ${ }^{2}$ Márcio de Castro Rodrigues ${ }^{3}$

Resumo: Este trabalho analisa a convergência absoluta e condicional da renda em Santa Catarina. Primeiramente, com base no PIB per capita, analisando a dependência espacial entre os 293 municípios do estado. Posteriormente, com base em microdados da PNAD, analisando a dinâmica da desigualdade entre gerações. $O$ modelo teórico provém de Solow (1956) e Jones (1997) e os instrumentos derivam das ferramentas da econometria espacial e da análise de dados em painel, utilizando as correções para amostras complexas. Os resultados mostram que há convergência de renda entre os municípios de Santa Catarina, podendo levar cerca de 20 anos para que a desigualdade se extinga. A análise espacial não apontou dependência espacial. Entre as gerações também há convergência, sendo fortemente impulsionada por políticas educacionais e de treinamento do trabalhador. Cada ano de estudo adicional acrescenta $20 \%$ no diferencial de renda e cada ano de experiência $7 \%$.

Palavras-chave: Convergência, Santa Catarina, PNAD

Abstract: This paper analyzes the absolute and conditional convergence of income in Santa Catarina. First, based on per capita GDP, analyzing spatial dependence among the 293 municipalities in the state. Subsequently, based on PNAD, analyzing the dynamics of inequality across generations. The theoretical model comes from Solow and Jones (1997) and instruments derived from the tools of spatial econometrics and the analysis of panel data, using corrections for complex samples. The results show that there is income convergence between the municipalities of Santa Catarina, but no spatial dependence. There are also intergenerational convergence being strongly driven by educational and worker training policies. Each additional year of study adds $20 \%$ and income differential in each year of experience $7 \%$.

Keywords: convergence, Santa Catarina, PNAD

JEL:R00

\section{INTRODUÇÃO}

A análise de convergência permite verificar se uma dada variável, como a renda, apresentando diferentes valores para as diferentes regiões ou

\footnotetext{
${ }^{1}$ Economista. Doutoranda em Economia Aplicada pela UFPE. Professora da Universidade Federal de Mato Grosso, campus Rondonópolis. krisley.mendes@uol.com.br

2 Economista. Doutorando em Economia Aplicada pela UFPE. Professor da Universidade Federal de Mato Grosso, campus Rondonópolis.f_nobuo@yahoo.com.br

3 Administrador. Especialista em gestão empresarial. Assessor Empresarial na Diretoria de Reestruturação de Ativos Operacionais do Banco do Brasil. marciocr@bb.com.br
} 
grupos, teria com o tempo uma diminuição nessa diferença, indicando que a desigualdade diminui. Portanto, permite analisar a dinâmica da desigualdade entre diferentes grupos ou regiões (LOPES, 2004).

Se há convergência, significa que a própria dinâmica econômica conduz à igualdade, prescindindo de políticas públicas voltadas para correções de desigualdade. Se não há convergência, seria necessário o estabelecimento de políticas adequadas à diminuição de desigualdade. Isso é bastante perigoso, pois a convergência pode ser verificada em níveis agregados, mas não entre grupos de pessoas, por idade, sexo ou cor. Além disso, a velocidade da convergência pode ser baixa, exigindo muitas décadas para que a desigualdade diminua. Assim, é importante que a análise de convergência seja feita em diferentes níveis de agregação e verificada sua velocidade para a definição de estabelecer ou não políticas sociais (MENEZES, SILVEIRA, AZZONI, 2006).

Esse assunto tem atraído a atenção de pesquisadores. Solow (1956) foi um dos precursores, fornecendo a base teórica para os estudos empíricos que lhe sucederam. Baumol (1986) estudou a convergência da renda de 16 economias industrializadas entre 1870 e 1879. Romer (1986) e Lucas (1988) apresentaram, nos anos 80, modelos de crescimento endógeno, acrescentando o capital humano (escolaridade e habilidades). Jones (1997) apresenta alternativas para estudos empíricos considerando as diferenças na velocidade de convergência. No Brasil, Menezes, Silveira e Azzoni (2006) investigam se a dinâmica das disparidades regionais pode ser influenciada por diferenças demográficas, abrindo alternativas para 0 estudo da convergência de renda entre grupos demográficos com a utilização de microdados.

O problema de pesquisa aqui investigado pergunta se existe convergência de renda absoluta e condicional entre os municípios e entre gerações no estado de Santa Catarina. Analisa, portanto, a convergência de renda entre os municípios de Santa Catarina, com base no PIB (Produto Interno Bruto) per capita entre 2001 e 2010, e entre as gerações, com base na renda pessoal extraída dos microdados da PNAD (Pesquisa Nacional por Amostra de Domicílios) entre 2002 e 2012.

Santa Catarina é o estado brasileiro que apresenta relativa homogeneidade na distribuição da renda e também o menor índice de desigualdade do país, sendo considerado um estado rico comparado aos demais da federação. Está inserido na região Sul do país, a qual também apresenta homogeneidade na dispersão do PIB per capita (RUSSO, SANTOS, PARRE, 2012). Isso, no entanto, não traduz as especificidades da desigualdade entre municípios e entre grupos. Assim, a motivação em estudar Santa Catarina está em verificar se essa homogeneidade também ocorre em níveis de agregação menores que o habitualmente considerado.

Considerando que o espaço importa nas decisões de firmas e trabalhadores, verificou-se a dependência espacial com os dados do PIB per capita. A disponibilidade de dados do PIB per capita para todos os municípios de Santa Catarina oportuniza analisar a dependência espacial da renda, auxiliando a elaboração de políticas públicas adequadas à dinâmica regional. 
Além disso, seguindo Menezes, Silveira Neto e Azzoni (2006) e considerando a importância da demografia na análise das desigualdades regionais, verificou-se a dinâmica da convergência de renda entre gerações, estabelecendo 14 coortes de idade, com os microdados da PNAD.

A amostra da PNAD é definida por conglomerados e estratos, com probabilidade variável de seleção, o que a define como amostra complexa. Assim, a amostra não é estruturada para produzir resultados para os diferentes níveis geográficos, impossibilitando a análise espacial com essa fonte de dados para municípios, meso ou microrregiões. Nesse estudo os microdados da PNAD são utilizados para a análise da demografia na dinâmica de convergência. $O$ tratamento para amostras complexas foi obtido de Skinner (1989), Silva, et al (2002) e Santos (2010), e se constitui numa das contribuições trazidas por esse trabalho, uma vez que tal tratamento tem sido ausente nos estudos de convergência que se utilizam dos dados da PNAD.

Além dessa introdução, o trabalho apresenta os materiais e métodos, no item 2, que descreve a obtenção das variáveis e apresenta os instrumentos econométricos utilizados, os procedimentos estatísticos e a base teórica do modelo. No item 3 são apresentados os resultados e discussão, onde também se compara com resultados de trabalhos empíricos anteriores. Por fim, as considerações finais.

\section{MATERIAIS E MÉTODOS}

\subsection{Materiais}

Para a análise espacial foram obtidos os dados do PIB e a população dos 293 municípios de Santa Catarina entre 2001 e 2010, fornecidos pelo IBGE (Instituto Brasileiro de Geografia e Estatística). Dividindo-se o PIB pela população obteve-se o PIB per capita por município, que será a variável dependente do modelo. O espaço amostral resultou em 2930 observações.

Para a análise demográfica foram extraídos dos microdados da PNAD (IBGE) os dados da renda de todas as fontes das pessoas acima de 10 anos, os anos de estudo e a idade (como proxy de experiência), entre 2002 e 2012, resultando em 98.860 observações. A idade é utilizada como proxy de experiência por Kuznets e Thomas (1957 apud CONNOLY, 2004) e na maioria de estudos empíricos depois dele, pois está associada à variável nãoobservada, ou seja, a experiência. $O$ grupo de pessoas acima de 10 anos deve-se à definição utilizada pelo IBGE para população em idade para trabalhar, ou seja, a População Economicamente Ativa (PEA). Estudo e experiência são variáveis indiretas para a inclusão do capital humano na análise de convergência. Uma importante discussão dessas proxys pode ser encontrada em Nakabashi e Figueiredo (2005).

A diferença do intervalo temporal para cada banco de dados (PIB e PNAD) se justifica pelo esforço em buscar o intervalo de 10 anos mais recente disponível. O PIB per capita municipal mais recente disponibilizado 
pelo IBGE até a elaboração desse trabalho era de 2010 e os microdados da PNAD mais recente eram o de 2012.

Os dados da PNAD foram tratados considerando a influência da amostra complexa. Essa influência não tem sido considerada na maioria dos estudos de convergência que utilizam os dados da PNAD, o que pode levar a resultados viesados. O tratamento de amostras complexas consiste, em suma, em agrupar unidades primárias de amostragem que apresentam uma única unidade amostral ${ }^{4}$, o que ajuda a produzir grandes variâncias. A desconsideração da influência de amostra complexa produz variâncias incorretas, dificultando a inferência dos resultados e a robustez de modelos (SKINNER, 1989; SILVA, et al, 2002; SANTOS, 2010).

O tratamento preliminar dos dados seguiu os 8 passos descritos abaixo:

1. Buscou-se primeiramente a extração dos dados de domicílios para a obtenção do desenho de amostragem (probabilidades regionais, estratos e unidades primárias de seleção - PSUs);

2. Em seguida foram extraídos os dados pessoais (sexo, idade, cor/raça, anos de estudo, renda principal, renda de todas as fontes, e peso);

3. Após fez-se a junção das informações do desenho da amostra, coletados de domicílios, com o arquivo de pessoas;

4. Em seguida, foi verificado se a junção dos dados ocorreu em todos os casos, excluindo-se os dados faltantes. Esses dados representaram entrevistas não realizadas nos domicílios, seja por recusa, domicílio fechado, entre outras razões;

5. Foi feita a declaração ao software de que se trata de amostra complexa (comando svyset no Stata), o que verifica a existência de estratos com uma única unidade de amostragem na fase 1 ;

6. Após foi feita a alocação de estratos com um único PSU em estratos com maior numero de observações (comando idonepsu do Stata);

7. Foi realizada a junção dos dados de cada ano num único arquivo, obtendo-se 98.860 observações para 10 anos de coleta;

8. Foram excluídas as observações com declaração de idade e anos de estudos indeterminados.

\subsection{Métodos}

\subsubsection{Base teórica do modelo}

\footnotetext{
${ }^{4}$ Ver em Silva, et al, 2002 maior detalhamento da construção da amostra da PNAD, a influência de amostras complexas na robustez de modelos e seu tratamento.
} 
O modelo provém de Solow, cujas hipóteses são: 1) existe apenas um bem na economia; 2 ) a poupança é uma função constante da renda ( $S=$ s. $Y)$; 3) a variação do estoque de capital $(\dot{K})$ é dada pelo investimento e a depreciação do capital; 4) a força de trabalho (L) cresce a uma taxa constante (n) igual à da taxa de crescimento da população; 5) função de produção $(Y)$ é homogênea de grau 1 (retornos constantes) e obedece à lei dos rendimentos decrescentes; 6) ambiente em concorrência perfeita. Esse é o modelo de Solow tradicional.

Para admitir crescimento é necessária uma hipótese adicional a respeito do progresso tecnológico (A). Como no modelo estamos em concorrência perfeita, admite-se que o progresso tecnológico é exógeno.

Com essas hipóteses temos a função de produção:

$$
Y=K^{\alpha} \cdot(A \cdot L)^{1-\alpha}
$$

Fazendo-se $\check{y}=\mathrm{Y} /($ A. L) e $\breve{\mathrm{k}}=\mathrm{K} /(\mathrm{A} \cdot \mathrm{L})$, tem-se o modelo:

$$
\begin{aligned}
& \check{y}=\breve{k}^{\alpha} \\
& \dot{L} / L=n \\
& \dot{K} / K=\frac{s Y}{K}-d \\
& \dot{A} / A=g
\end{aligned}
$$

Tomando-se $\breve{k}=K /(A . L)$, aplicando logaritmo e derivando em relação ao tempo temos que:

$$
\frac{\dot{\mathrm{k}}}{\overline{\mathrm{k}}}=\frac{\mathrm{s}, \breve{y}}{\breve{\mathrm{k}}}-(\mathrm{n}+\mathrm{g}+\mathrm{d})
$$

Ou seja, a dinâmica de crescimento do capital depende da taxa de poupança, do crescimento populacional, do progresso tecnológico e da taxa de depreciação.

No estado estacionário, em equilíbrio, $\dot{\mathrm{k}} / \breve{\mathrm{k}}=0$, ou seja, o crescimento de capital é constante. Logo o crescimento dependerá da taxa de crescimento populacional, do progresso tecnológico e da depreciação, que serão constantes: 


$$
\frac{\mathrm{s}, \breve{y}}{\widetilde{\mathrm{k}}}=(\mathrm{n}+\mathrm{g}+\mathrm{d})
$$

E a função de produção resulta em:

$$
Y^{*}=A \cdot(s / n+g+d)^{\frac{\alpha}{1-\alpha}}
$$

Onde o sobrescrito * indica a variável no estado estacionário. O país considerado mais rico é aquele que apresenta o maior produto per capita. Quanto maior a taxa de poupança do país, maior será sua taxa de crescimento. No estado estacionário todos os países crescem a mesma taxa, porém seu produto per capita dependerá das suas condições iniciais.

A taxa de crescimento dependerá somente do progresso tecnológico, uma vez que $(s / n+g+d)$ será constante. Chamando $(s / n+g+d)$ de "a", temos:

$$
\begin{aligned}
\ln Y^{*} & =\ln A+\ln a \\
\frac{d \ln Y^{*}}{d t} & =\frac{d \ln A}{d t}+\frac{d \ln a}{d t}
\end{aligned}
$$

Como "a" é constante $\frac{d l n a}{d t}=0$, então a taxa de crescimento do produto no tempo depende do progresso tecnológico $A$.

Para países com mesmas condições iniciais o produto de estado estacionário converge em nível, ou seja, seus produtos serão iguais, o que se define por convergência absoluta. $O$ modelo de Solow prevê a convergência em taxa, que é chamada convergência condicional: países crescerão à mesma taxa, mas seus produtos serão diferentes por suas condições iniciais. Se há dinâmica de convergência condicional cada região precisa de uma política diferente.

A velocidade de crescimento de cada país ou região dependerá da distância que cada um estiver de seu estado estacionário. Um país próximo de seu estado estacionário cresce a uma taxa menor que outro que esteja distante do seu estado estacionário (EE). As implicações em termos de política é definir qual variável pode ser afetada por um choque de modo a fazer o país que esteja próximo de seu EE retomar o crescimento, ou postergar sua estacionaridade. Pelo modelo, essa variável seria a poupança, única passível de ser afetada por política pública, como choques em fundo de pensão entre outras.

A velocidade com que a economia atinge o estado estacionário é importante do ponto de vista de políticas. É dada por uma proporção da diferença entre o produto corrente e o produto do estado estacionário. 


$$
\frac{d \ln Y^{*}}{d t}=-\beta\left(\ln \check{y}_{t}-\ln \check{y}^{*}\right)
$$

Onde $\beta$ é a velocidade de convergência. Para obtê-la, toma-se o modelo a partir da função de produção e após linearizações e derivações chega-se à equação de convergência ${ }^{5}$ :

$$
\Delta \ln y_{t}=\left(1+\mathrm{e}^{-\beta t}\right) \cdot \ln A_{0}+\left(1+\mathrm{e}^{-\beta t}\right) \cdot \ln a-\left(1+\mathrm{e}^{-\beta t}\right) \cdot \ln y_{0}+g_{t}
$$

Onde: $\left(1+\mathrm{e}^{-\beta t}\right) \cdot \ln A_{0}+\left(1+\mathrm{e}^{-\beta t}\right) \cdot=\lambda_{0} ; \quad-\left(1+\mathrm{e}^{-\beta t}\right)=\lambda_{1} ;$ e $g_{t} 0$ erro estocástico. A equação para regressão resulta:

$$
\Delta \ln y_{t}=\lambda_{0}+\lambda_{1} \cdot \ln y_{0}+g_{t}
$$

Ao se obter $\lambda_{1}$ resolve-se $-\left(1+\mathrm{e}^{-\beta t}\right)$, conhecendo-se a velocidade de convergência $\beta$. Se $\lambda_{1}$ é significativo, diz-se que há convergência absoluta. Se não há convergência pode-se incluir mais variáveis explicativas no modelo de forma a obter convergência, porém estará condicionada a essas variáveis adicionais (JONES, 1997; REY, 1999) .

Logo, algo importante a se responder é se há convergência absoluta ou condicional. $\mathrm{O}$ que se acrescenta à condicional são variáveis como estudos e experiência, por exemplo. Se essas variáveis alteram a velocidade de convergência passam a oferecer mais opções de política além da poupança. Assim podemos ter, como sugere Jones:

$$
\Delta \ln y_{t}=\left(1+\mathrm{e}^{-\beta t}\right) \cdot \ln A_{0}+\left(1+\mathrm{e}^{-\beta t}\right) \cdot \ln a-\left(1+\mathrm{e}^{-\beta t}\right) \cdot \ln y_{0}+\left(1+\mathrm{e}^{-\beta t}\right) \cdot \ln Z g_{t}
$$

Onde Z é um vetor de variáveis condicionais. Assim, pode-se fazer:

$$
\Delta \ln y_{t}=\lambda_{0}+\lambda_{1} \cdot \ln y_{0}+\lambda_{2} E d u c+\lambda_{3} \text { Idade }+\lambda_{3} E d u c^{2}+g_{t}
$$

\subsubsection{Procedimento Estatístico}

Este trabalho irá, portanto, estimar a convergência absoluta:

\footnotetext{
${ }^{5}$ A demonstração pode ser obtida em Barro e Sala-i-Martin (1995)
} 


$$
\Delta \ln y_{t}=\lambda_{0}+\lambda_{1} \cdot \ln y_{0}+g_{t}
$$

Onde: $y_{t}=$ PIBpercapita $; y_{0}=$ PIB per capita inicial do período.

Esse modelo será estimado com dados do PIB per capita, testando-se os resultados da estimação em cross-section, por OLS (Mínimos Quadrados Ordinários), e em painel, pelo modelo de efeitos fixos e aleatórios. Serão utilizados os instrumentos da econometria espacial para testar a dependência espacial da renda calculando-se I-Moran, LM Robusto elaborando-se mapas e gráficos que atestem os resultados.

A convergência condicional será avaliada estimando-se:

$$
\Delta \ln y_{t}=\lambda_{0}+\lambda_{1} \cdot \ln y_{0}+\lambda_{2} E d u c+\lambda_{3} \text { Idade }+\lambda_{3} E d u c^{2}+g_{t}
$$

Onde: $y_{t}=$ renda de todas as fontes; $y_{0}=$ renda de todas as fontes com defasagens; $E d u c$ = anos de estudos; e ldade como proxy de experiência.

Essa estimação será realizada com os microdados da PNAD, sendo os dados primeiramente tratados para considerar a influência da amostra complexa. Como a amostra da PNAD não é elaborada para oferecer resultados a diferentes níveis geográficos, não é possível avaliar a dependência espacial dos municípios a partir dessa fonte de dados.

Os modelos foram estimados com o auxílio do software STATA 11.1.

\section{RESULTADOS E DISCUSSÃO}

\subsection{Convergência Absoluta em Santa Catarina}

A Tabela 1 abaixo apresenta os resultados da estimação $\Delta \ln y_{t}=\lambda_{0}+$ $\lambda_{1} \cdot \ln y_{0}+g_{t}$, em cross-section (onde a variável explicativa $y_{0}$ é o PIB per capita inicial) e em painel (onde a variável explicativa $y_{0}$ é o PIB per capita com 1 defasagem).

Os resultados apontam para a existência de convergência absoluta entre os municípios de Santa Catarina nos 2 modelos estimados.

Tabela 1: Estimativa dos parâmetros de análise de convergência do PIB per capita dos municípios de Santa Catarina - 2001 a 2010

\begin{tabular}{ccc|cc}
\hline Parâmetros & Cross-Section & $\beta$ - velocidade & $\begin{array}{c}\text { Dados em } \\
\text { Painel }\end{array}$ & $\beta$ - velocidade \\
\hline$\lambda_{1}$ & $-0,313272$ & 0,03758 & $-0,5248022$ & 0,074402 \\
$\rho$-valor & 0,000 & & 0,000 &
\end{tabular}




\begin{tabular}{cc|c}
$\lambda_{0}$ & 2,981472 & 4,260099 \\
$\rho$-valor & 0,000 & 0,000
\end{tabular}

Fonte: Elaborado pelos autores.

A significância de $\lambda_{1}$ indica a existência de convergência absoluta. Significa que há uma tendência dos municípios mais pobres crescerem mais rápido do que os mais ricos, conduzindo à diminuição da desigualdade. $\mathrm{A}$ velocidade com que isso ocorre é dada por $\beta$ - velocidade. Como o produto no estado estacionário não é observado, calcula-se o tempo que levaria para percorrer metade do caminho. Assim:

$$
\begin{gathered}
\frac{1}{2}=-\left(1-\mathrm{e}^{-\beta t}\right. \\
-\frac{1}{2}=-\mathrm{e}^{-\beta t} \\
-\beta t=\ln \frac{1}{2} \\
\beta t=0,693 \\
t=0,693 / \beta)
\end{gathered}
$$

Com a velocidade obtida em cross-section, ou seja levando em conta as condições iniciais do período, o tempo que levaria para percorrer metade do caminho para o estado estacionário seria cerca de 18 anos, ou cerca de 40 anos no total.

Uma importante limitação do cross-section é não assumir as especificidades dos municípios. A capacidade de crescimento de cada um não é igual, isso seria captado por efeitos fixos, possíveis de serem inseridos em dados em painel. Para trabalhar em painel e manter a representatividade dos municípios utilizou-se a diferença de apenas 1 ano do logaritmo do PIB per capita em relação à sua defasagem ao ano imediatamente anterior.

Com a velocidade obtida por dados em painel, ou seja, levando em conta as condições do período imediatamente anterior, o tempo para percorrer metade do caminho do estado estacionário seria cerca de 9 anos. Assim, a desigualdade entre municípios catarinenses seria superada em cerca de 20 anos no total.

\subsection{Convergência espacial em Santa Catarina}

O mapa 1 apresenta a diferença do logaritmo do PIB per capita em relação ao produto inicial, que representa a taxa de crescimento do produto per capita. Não se vê claramente a formação de clusters significativos. Salvo na região norte do meio oeste catarinense e uma região de baixo 
desenvolvimento que vai do meio oeste sul em direção a noroeste. Alguns clusters formados por 3 a 4 municípios distribuídos em todo o estado.

Figura 1: Mapa de Santa Catariana com a taxa de crescimento do produto per capita dos municípios entre 2001 e 2010

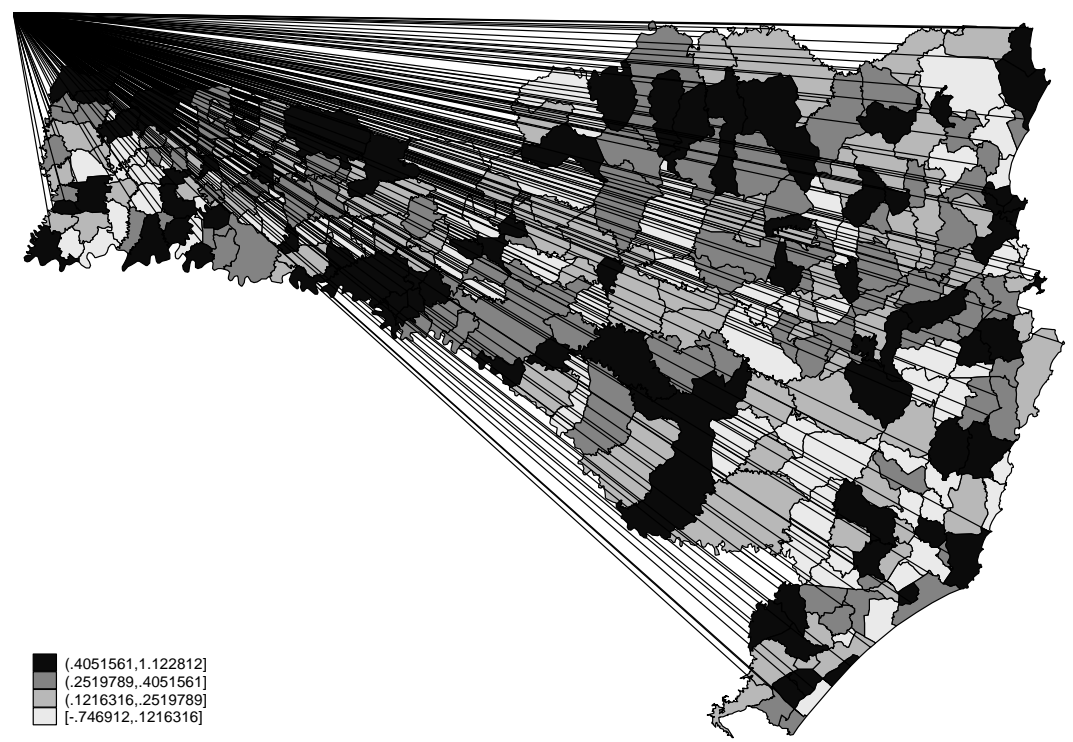

Fonte: Elaboração própria com dados do IBGE e auxílio do software Stata 11.1.

O Mapa 2 apresenta o produto inicial dos municípios. A hipótese de convergência ganha força na comparação com o mapa anterior, pois em geral as cores estão invertidas, indicando que municípios de baixo PIB per capita inicial (Mapa 2) apresentam altas taxas de crescimento (Mapa 1).

Figura 2: Mapa de Santa Catarina com o Produto Interno Bruto per capita no período inicial dos municípios - 2001 


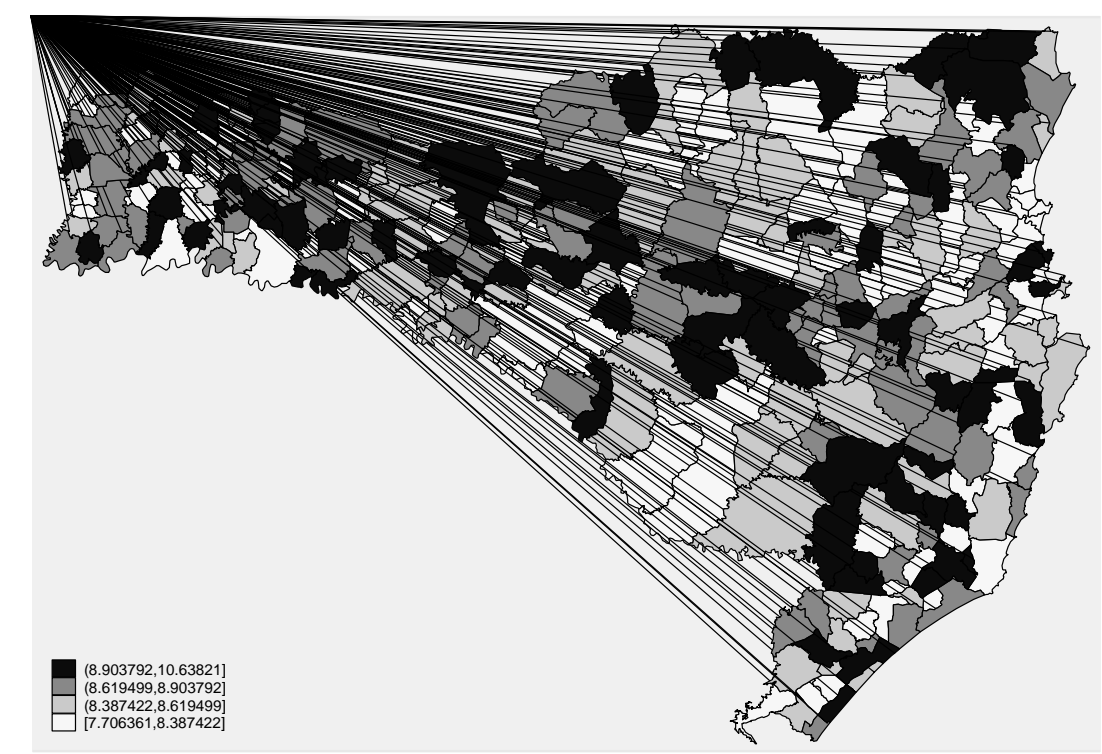

Fonte: Elaboração própria com dados do IBGE e auxílio do software Stata 11.1.

Para estabelecer a matriz de vizinhança, foi construído o parâmetro subtraindo longitude maior da menor e a latitude maior da menor, definindo com isso o diâmetro médio das distâncias.

Tabela 2: Latitude e Longitude de Santa Catarina

\begin{tabular}{|c|c|c|c|c|c|c|}
\hline Variáveis & Obs. & Média & $\begin{array}{l}\text { Desvio } \\
\text { Padrão }\end{array}$ & Min. & Max. & Diferença \\
\hline $\begin{array}{l}\text { X_stub_ } \\
\text { (Latitude) }\end{array}$ & 293 & 323.3479 & 160.8004 & 28.70695 & 542.9774 & 514,2705 \\
\hline $\begin{array}{l}\text { y_stub_- } \\
\text { (Longitude) }\end{array}$ & 293 & -3021.5 & 80.83533 & 3246.503 & -2893.197 & 353,3060 \\
\hline $\begin{array}{l}\text { Diâmetro } \\
\text { médio }\end{array}$ & & & & & & 623,9385 \\
\hline
\end{tabular}

Fonte: IBGE, 2013.

Foi verificada que a maior distância mínima entre vizinhos é de 32,42 e a menor distância máxima é de 275,18. A matriz de vizinhança foi estabelecida definindo-se a banda 40 , que está entre os dois intervalos. A definição de vizinho é o maior desafio dessa literatura, pois em suma o estabelecimento da banda ocorre por tentativa e erro, por exemplo: sob banda 10 não foi possível criar a matriz, sob banda 30 duas localizações ficaram sem vizinhos, apenas na banda 40 foi criada a matriz de vizinhança. Uma importante discussão a respeito das medidas de ponderação espacial é feita em TYSZLER.

O I-Moran é a estatística que calcula a correlação entre a renda de dada localização com a renda do vizinho, identificando a correlação espacial. Essa estatística varia entre -1 e 1 e fornece uma medida de associação linear entre os vetores $Z_{t}$ e a média ponderada dos valores da vizinhança $\left(W Z_{t}\right)$. O IMoran tem um valor esperado de $-[1 /(n-1)]$, de modo que valores próximos de zero indicam a ausência de um padrão espacial dos dados, ou seja, a ausência de correlação espacial. Para Santa Catarina, com os dados do PIB per capita temos: 
Tabela 3:Correlograma Espacial - I-Moran

\begin{tabular}{lccccc}
\hline Distancebands & $\mathbf{I}$ & $\mathbf{E}(\mathbf{l})$ & $\mathbf{s d}(\mathbf{I})$ & $\mathbf{z}$ & p-value $^{*}$ \\
\hline$(0-5]$ & - & -0.003 & - & $\dot{ }$ & $\dot{0}$ \\
$(0-10]$ & 0.193 & -0.003 & 0.134 & 1.471 & 0.071 \\
$(0-15]$ & 0.064 & -0.003 & 0.067 & 1.009 & 0.157 \\
$(0-20]$ & 0.031 & -0.003 & 0.048 & 0.727 & 0.234 \\
$(0-25]$ & 0.024 & -0.003 & 0.038 & 0.715 & 0.237 \\
$(0-30]$ & 0.041 & -0.003 & 0.031 & 1.427 & 0.077 \\
$(0-35]$ & 0.024 & -0.003 & 0.027 & 1.045 & 0.148 \\
$(0-40]$ & 0.013 & -0.003 & 0.023 & 0.716 & 0.237 \\
\hline
\end{tabular}

Fonte: Calculado a partir de dados do IBGE com o auxílio do software Stata 11.1

Pela tabela vê-se que a hipótese de correlação espacial só seria aceitável a partir de $7,1 \%$ de significância. Isso indica que, considerando ausência de correlação espacial, os parâmetros de convergência calculados acima não são viesados. Para verificar isso graficamente foi elaborado 0 gráfico do teste de Moran para a taxa de crescimento do PIB per capita (à esquerda) e para o PIB per capita no período inicial (à direita).

Figura 3. Gráfico do teste de Moran para a taxa de crescimento do PIB per capita (à esquerda) e para o PIB per capita no período inicial (à direita)
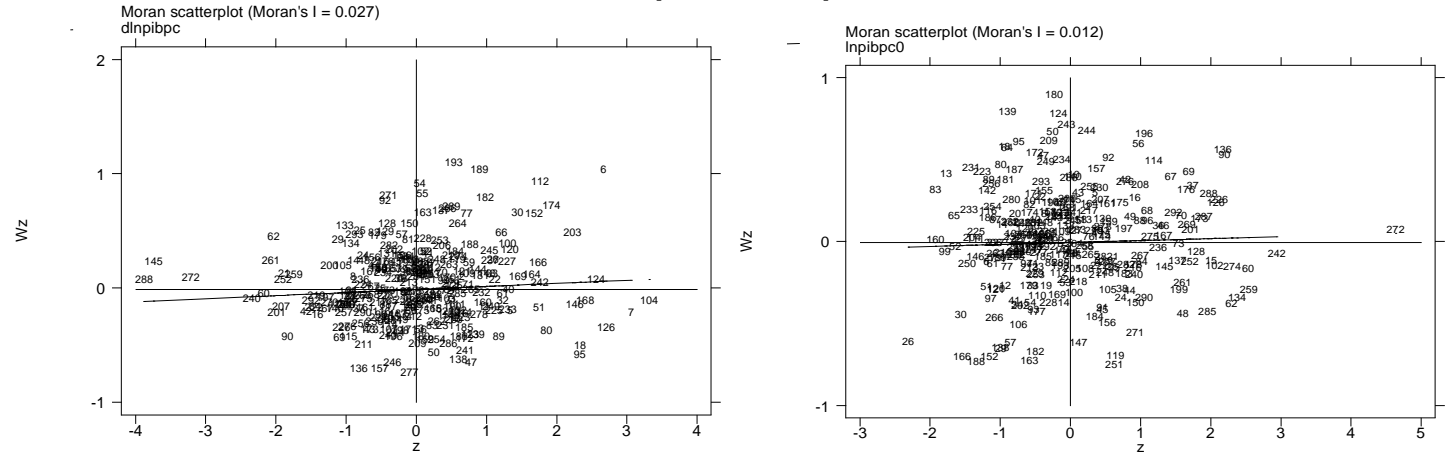

Fonte: Elaboração própria com dados do IBGE e auxílio do software Stata 11.1.

A maior concentração de municípios em torno do eixo zero confirma a ausência de correlação espacial, havendo correlação do tipo Alto-Alto (AA) para cerca de 10 municípios distribuídos no canto superior direito do gráfico à esquerda, outros 9 municípios do tipo Alto-Baixo (AB) concentrados no canto inferior direito, 7 do tipo Baixo-Baixo (BB) e 5 do tipo Baixo-Alto. Considerando que o estado é formado por 293 municípios, esse nível de correlação não justificaria o uso de modelos espaciais para atestar a convergência de renda no estado.

Os municípios que apresentaram correlação espacial significativa da taxa de crescimento são apresentados no quadro abaixo por tipo de correlação. O quadro inspira avaliar a convergência da mesorregião do Oeste Catarinense isoladamente.

\section{Quadro 1: Municípios e suas mesorregiões que apresentaram correlação espacial significativa por tipo de correlação ${ }^{6}$}

\footnotetext{
${ }^{6}$ Alto-Alto: municípios de alto desenvolvimento cercados por municípios de alto desenvolvimento; AltoBaixo: municípios de alto desenvolvimento cercados por municípios de baixo desenvolvimento; Baixo-
} 


\begin{tabular}{|c|c|c|c|}
\hline $\begin{array}{l}\text { Alto -Alto } \\
\text { Município }\end{array}$ & Mesorregião & $\begin{array}{l}\text { Alto-Baixo } \\
\text { Município }\end{array}$ & Mesorregião \\
\hline Águas de Chapecó & Oeste Catarinense & Calmom & Oeste Catarinense \\
\hline Novo Horizonte & Oeste Catarinense & Lebon Regis & Oeste Catarinense \\
\hline Itá & Oeste Catarinense & Guatambú & Oeste Catarinense \\
\hline Palma Sola & Oeste Catarinense & Águas Frias & Oeste Catarinense \\
\hline Macieira & Oeste Catarinense & $\begin{array}{l}\text { Dionísio } \\
\text { Arqueiro }\end{array}$ & Oeste Catarinense \\
\hline Bandeirante & Oeste Catarinense & Araquari & Oeste Catarinense \\
\hline Petrolância & Vale do Itajaí & Itajaí & Vale do Itajaí \\
\hline Porto Belo & $\begin{array}{l}\text { Vale do Itajaí } \\
\text { Grande }\end{array}$ & Morro Grande & Sul Catarinense \\
\hline $\begin{array}{l}\text { Paulo Lopes } \\
\text { Imbituba }\end{array}$ & $\begin{array}{l}\text { Florianópolis } \\
\text { Sul Catarinense }\end{array}$ & Garuva & Norte Catarinense \\
\hline
\end{tabular}

Fonte: Elaborada pelo autor.

Quadro 1: Municípios e suas mesorregiões que apresentaram correlação espacial significativa por tipo de correlação (continuação).

\begin{tabular}{|c|c|c|c|}
\hline Baix & Baixo & & xo-Alto \\
\hline Município & Mesorregião & Município & Mesorregião \\
\hline Joaçaba & Oeste Catarinense & Catanduvas & Oeste Catarinense \\
\hline Maravilha & Oeste Catarinense & Tangará & Oeste Catarinense \\
\hline Tunápolis & Oeste Catarinense & Vitor Meirelles & Vale do Itajaí \\
\hline Quilombo & Oeste Catarinense & Lauro Muller & Sul Catarinense \\
\hline Presidente Castelo & Oeste Catarinense & & Sul Catarinense \\
\hline São João do Sul & Sul Catarinense & & \\
\hline Forquilhinha & Sul Catarinense & & \\
\hline Ponte Alta do Norte & Serrana & & \\
\hline
\end{tabular}

Para atestar a ausência de correlação espacial, foi verificada pelo teste de Moran a ausência de autocorrelação dos resíduos da regressão, cujos resultados foram apresentados na Tabela 1. A concentração dos resíduos em torno do eixo zero atesta que a regressão produz parâmetros não visados.

Figura 4. Gráfico do teste de Moran dos resíduos da regressão

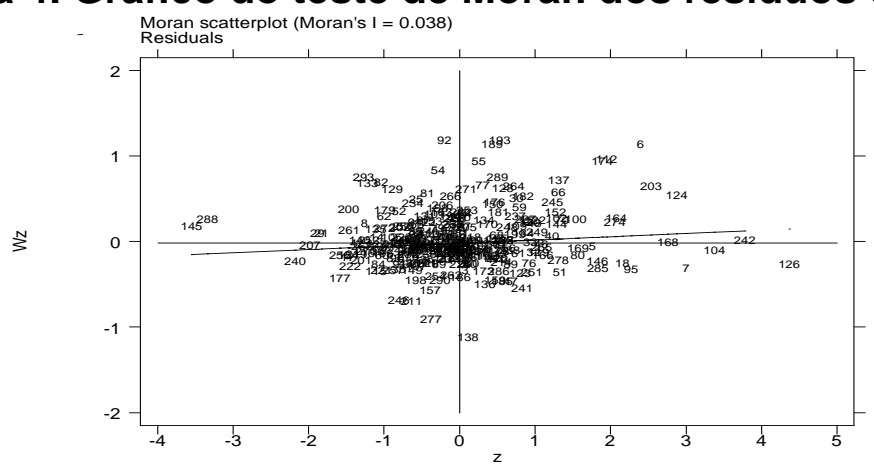

Fonte: Elaboração própria com dados do IBGE e auxílio do software Stata 11.1.

Baixo: municípios de baixo desenvolvimento cercados por municípios de baixo desenvolvimento; BaixoAlto: municípios de baixo desenvolvimento cercados por municípios de alto desenvolvimento 
Testes adicionais confirmam a ausência de dependência espacial na regressão realizada por OLS. Além da estatística I-Moran, o Multiplicador de Lagrange e 0 Multiplicador de Lagrange Robusto não apresentaram significância abaixo de $5 \%$, tanto para o teste de erro espacial, quanto para 0 teste de lag espacial.

\section{Tabela 4: Testes diagnósticos para dependência espacial na regressão por OLS}

\begin{tabular}{lccc}
\hline Test & Statistic & DF & p-value \\
\hline Spatialerror: & & & \\
Moran's I & 1.658 & 1 & 0.097 \\
Lagrangemultiplier & 2.217 & 1 & 0.137 \\
RobustLagrangemultiplier & 1.372 & 1 & 0.241 \\
Spatiallag: & & & \\
Lagrangemultiplier & 1.275 & 1 & 0.259 \\
RobustLagrangemultiplier & 0.430 & 1 & 0.512 \\
\hline
\end{tabular}

Fonte: calculado a partir de dados do IBGE com o auxílio do software Stata 11.1

\subsection{Convergência na Renda Pessoal em Santa Catarina: Análise por Coorte de Idade}

A análise seguinte avalia a convergência em nível menor de agregação, com base nos microdados da PNAD, utilizando dados da renda pessoal, escolaridade e idade das pessoas maiores de 10 anos.

Os dados foram agrupados em 14 coortes de idade para o intervalo entre 87 e 17 anos, cada coorte com amplitude de 5 anos, conforme tabela abaixo:

\begin{tabular}{|c|c|c|c|}
\hline Coortes & $\begin{array}{l}\text { Amplitude } \\
\text { nascimento }\end{array}$ & de ano & de Amplitude da idade \\
\hline coorte $=1$ & $>1925$ & $<=1929$ & 87 a 83 anos \\
\hline coorte $=2$ & $>=1930$ & $<=1934$ & 82 a 78 anos \\
\hline coorte $=3$ & $>=1935$ & $<=1939$ & 77 a 73 anos \\
\hline coorte $=4$ & $>=1940$ & $<=1944$ & 72 a 68 anos \\
\hline coorte $=5$ & $>=1945$ & $<=1949$ & 67 a 63 anos \\
\hline coorte $=6$ & $>=1950$ & $<=1954$ & 62 a 58 anos \\
\hline coorte $=7$ & $>=1955$ & $<=1959$ & 57 a 53 anos \\
\hline coorte $=8$ & $>=1960$ & $<=1964$ & 52 a 48 anos \\
\hline coorte $=9$ & $>=1965$ & $<=1969$ & 47 a 43 anos \\
\hline coorte $=10$ & $>=1970$ & $<=1974$ & 42 a 38 anos \\
\hline coorte $=11$ & $>=1975$ & $<=1979$ & 37 a 33 anos \\
\hline coorte $=12$ & $>=1980$ & $<=1984$ & 32 a 28 anos \\
\hline coorte $=13$ & $>=1985$ & $<=1989$ & 27 a 23 anos \\
\hline coorte $=14$ & $>=1990$ & $<=1995$ & 22 a 17 anos \\
\hline
\end{tabular}

Fonte: Elaborada pelo autor. 
Com isso descartou-se 17.725 observações de pessoas com idades fora desse intervalo. Também verificou-se que 22.040 das pessoas remanescentes não declararam renda, excluindo-se essas observações nessa variável. Ao agrupar os dados por coorte anualmente gera-se a média de renda para cada coorte a cada um dos 10 anos coletados. Com isso a regressão trabalha com 140 observações.

A variável dependente é criada gerando-se a diferença do logaritmo neperiano da renda de todas as fontes em relação ao período anterior. Essa variável será regredida em relação às variáveis explicativas: renda pessoal de todas as fontes, média dos anos de estudos de cada coorte em cada ano, idade média de cada coorte em cada ano, e o quadrado da idade média, todos com 1 defasagem. Foram estimados 6 modelos, alternando-se a presença de dummies temporal e para coorte. As dummies apresentaram significância apenas nos modelos (2), (6) e (7).

Os resultados são apresentados na tabela a seguir:

\begin{tabular}{|c|c|c|c|c|c|c|c|c|}
\hline \multirow{2}{*}{ Parâmetros } & \multicolumn{4}{|c|}{ Convergência Absoluta } & \multicolumn{4}{|c|}{ Convergência Condicional } \\
\hline & (1) & (2) & (3) & (4) & (5) & (6) & (7) & (8) \\
\hline Const & $\begin{array}{l}0,82578 \\
(0.000)\end{array}$ & $\begin{array}{l}1,56849 \\
(0.000)\end{array}$ & $\begin{array}{l}2,03278 \\
(0.000)\end{array}$ & $\begin{array}{l}0,69044 \\
0.011\end{array}$ & \begin{tabular}{|l|}
0,82840 \\
$(0.000)$
\end{tabular} & $\begin{array}{l}3,54296 \\
(0.000)\end{array}$ & $\begin{array}{l}29,06090 \\
0.148\end{array}$ & $\begin{array}{l}1,76435 \\
(0.000)\end{array}$ \\
\hline Iny1 & $\begin{array}{l}0,09817 \\
(0.004)\end{array}$ & $\begin{array}{l}0,18916 \\
(0.000)\end{array}$ & $\begin{array}{l}0,35367 \\
(0.000)\end{array}$ & $\begin{array}{l}0,05944 \\
0.218\end{array}$ & $\begin{array}{l}-0,22754 \\
0.005\end{array}$ & $\begin{array}{l}-0,93803 \\
(0.000)\end{array}$ & $\begin{array}{l}-1,16365 \\
(0.000)\end{array}$ & $\begin{array}{l}-1,04421 \\
(0.000)\end{array}$ \\
\hline Anos & & & & & & & & \\
\hline Estudo & & & & & $\begin{array}{l}0,08843 \\
0.007\end{array}$ & $\begin{array}{l}0,19748 \\
(0.000)\end{array}$ & $\begin{array}{l}0,34341 \\
(0.000)\end{array}$ & $\begin{array}{l}0,33513 \\
(0.000)\end{array}$ \\
\hline Idade & & & & & $\begin{array}{l}-0,00063 \\
0.940\end{array}$ & $\begin{array}{l}0,06839 \\
(0.000)\end{array}$ & $\begin{array}{l}-0,23044 \\
0.341\end{array}$ & $\begin{array}{l}0,13281 \\
(0.000)\end{array}$ \\
\hline Idade 2 & & & & & $\begin{array}{l}0,00008 \\
0.285\end{array}$ & $\begin{array}{l}-0,00048 \\
(0.000)\end{array}$ & $\begin{array}{l}-0,00042 \\
0.029\end{array}$ & $\begin{array}{l}-0,00014 \\
0.465\end{array}$ \\
\hline $\begin{array}{l}\text { Dummy } \\
\text { temporal }\end{array}$ & Não & $\operatorname{sim}$ & $\operatorname{sim}$ & não & não & $\operatorname{sim}$ & $\operatorname{sim}$ & não \\
\hline $\begin{array}{l}\text { Dummy } \\
\text { para coortes }\end{array}$ & Não & não & $\operatorname{sim}$ & $\operatorname{sim}$ & não & não & $\operatorname{sim}$ & $\operatorname{sim}$ \\
\hline$\beta-$ & & & & & & & & \\
\hline Velocidade & $-0,0103$ & $-0,021$ & $-0,0436$ & - & $-0,02582$ & $-0,2781$ & - & - \\
\hline $\mathrm{R} 2$ & 0.0648 & 0.2439 & 0.3162 & 0.1134 & 0.1493 & 0.4990 & 0.6072 & 0.4748 \\
\hline N. Obs & 126 & 126 & 126 & 126 & 126 & 126 & 126 & 126 \\
\hline
\end{tabular}

Fonte: estimativas dos autores a partir de dados da PNAD (IBGE). ${ }^{*}$ os valores entre parênteses são os $\rho$-valor dos parâmetros.

As dummies apresentaram significância apenas nos modelos (2), (6) e (7). No modelo (7) a constante não apresentou significância estatística, o que não faria sentido econômico, uma vez que no modelo de Solow ela representa a taxa de variação constante do produto formada pelo crescimento populacional, progresso tecnológico e depreciação do capital. Assim, são consistentes os modelos (2) e (6). O modelo (4), único a apontar ausência de convergência, também não apresentou significância para nenhuma das 
dummies para coortes, no entanto o modelo (1) que não tem a dummy apresenta convergência com $0,4 \%$ de significância.

São, portanto, sobreviventes os modelos (1) e (2) de convergência absoluta e (5) e (6) de convergência condicional. Todos confirmam a convergência de renda entre coortes. Os modelos (2) e (6) apresentam maior poder preditivo pelo valor do $\mathrm{R}^{2}$, além de controlarem efeitos aleatórios temporais.

Pelo modelo (2) observa-se a existência de convergência absoluta da renda no estado entre coortes, mas seria atingida em cerca de 67 anos ( $\beta$ velocidade de 0,021).

No modelo $(6)$, o coeficiente de Inw $1(-0,938)$ indica haver convergência de renda no estado entre as gerações, com $\beta$-velocidade para 10 anos de 0,278104 . Com essa velocidade de convergência, o estado estacionário entre gerações seria atingido em cerca de 5 anos. A convergência aqui é impulsionada pela educação e pela experiência.

O coeficiente da variável anos de estudo (educ1) indica que para cada 1 ano de estudo adicional há um ganho de cerca de $20 \%$ no diferencial de renda entre um período e outro. O parâmetro estimado da média de idade (id1 $=0,068$ ) indica que cada ano adicional de experiência oferece um ganho médio de cerca e $7 \%$ no diferencial de renda. Isso indica que políticas voltadas para a manutenção das pessoas na escola e o treinamento ao trabalho aceleram a velocidade de convergência, diminuindo em 62 anos o tempo de redução da desigualdade pelo mercado.

\section{CONSIDERAÇÕES FINAIS}

Este trabalho apresentou análise de convergência de renda no estado de Santa Catarina no período de 2001 a 2012. Primeiramente, utilizando dados agregados do PIB per capita dos municípios, depois utilizando microdados da PNAD. Os resultados atestam a equivalência de se trabalhar com microdados no lugar de dados agregados para a análise de convergência, o que possibilita pormenorizar a análise com coortes demográficos, como já demonstrado por Menezes, Silveira Neto e Azzoni (2006). O inconveniente seria a impossibilidade de trabalhar a análise espacial em níveis geográficos menores que o nacional, já que a PNAD não produz resultados para municípios, meso ou microrregiões.

Os resultados apontam para a existência de convergência absoluta do PIB per capita municipal no estado, podendo levar cerca de 20 anos para que a desigualdade se extinga entre os municípios. A análise espacial constatou que não há dependência espacial na regressão realizada por OLS e que, portanto, os parâmetros de convergência estimados são não viesados. No entanto, entre os 32 municípios que apresentaram significância estatística na dependência espacial, $60 \%$ são da mesorregião oeste catarinense, o que inspira uma análise pormenorizada dessa região a trabalhos futuros.

A análise com microdados da PNAD, utilizando a renda pessoal como variável, também apresentou convergência absoluta, mas 0 estado 
estacionário seria atingido em 67 anos. A convergência condicional é avaliada acrescentando-se os variáveis anos de estudo e idade, como proxy de experiência. A convergência se mantém, com velocidade maior, sendo necessários 5 anos para a extinção da desigualdade. Cada ano adicional de estudo acrescenta cerca de $20 \%$ no ganho de renda e cada ano a mais de experiência acelera o ganho de renda em $7 \%$. A convergência é então fortemente impulsionada por políticas educacionais e de treinamento do trabalhador.

\section{REFERÊNCIAS}

BARRETO, R. C. S. \& ALMEIDA, E. S. \& LIMA, J. E. (2010) Convergência espacial do PIB.per capita no estado do Ceará. Revista de Economia, v. 36, n. 3 (ano 34), p. 25-40, set./dez 2010. Editora UFPR

BARRO, R.J., AND X. SALA-I-MARTIN (1995). Economic Growth. McGreawHill, New York..

BAUMOL, W. J.(1986) Productivity growth, convergence, and welfare: What the long-run data show. American Economic Review, v. 76, n. 5, p. 1072-85, December.

CONNOLLY, M. P. 2004. Human Capital and Growth in the Post-Bellum South: A Separate but Unequal Story. Journal of Economic History, 64(2): 1-39.

JONES, C. (1997), "On the Evolution of the World Income Distribution," Journal of Economic Perspectives 11, 19-36.

LOPES, J. L. Avaliação do processo de convergência da produtividade da terra na agricultura brasileira no período de 1960 a 2001. Tese de Doutorado, ESALQ/USP, 2004. (mimeo).

LUCAS, R. E. (1988). On the mechanics of Economic development. Journal of Monetary Economics. v.22, n. 1, p. 3- 42.

MENEZES, T. A.; SILVEIRA NETO, R. da M.; AZZONI, C. R. (2006) Demografia, ciclo de vida e dinâmica da desigualdade regional de renda no Brasil. Anais: XXXIV Encontro Nacional de Economia. Disponível em: http://www.anpec.org.br/encontro2006/artigos/A06A028.pdf

NAKABASHI, L. FIGUEIREDO, L. Capital Humano: Uma Nova Proxy Para Incluir Aspectos Qualitativos. Belo Horizonte: UFMG/Cedeplar, 2005. 36p. (Texto para discussão ; 270).

PEROBELLI, F. S.; FARIA, W. R.; FERREIRA, P. G. C.. Análise de Convergência Espacial do PIB Per Capita em Minas Gerais: 1975-2003. Fórum: Banco do Nordeste. Disponível em: http://www.bnb.gov.br/content/aplicacao/eventos/forumbnb2006/docs/analise_ de_convergencia.pdf

REY, S \& MONTOURI, B. (1999). US Regional Income Convergence: a spatial econometric perspective, Regional Studies, n 33, p. 146-156

ROMER, P.M. (1986) Increasing returns and long run growth. Journal of Political Economy, v.94, n.5. 
RUSSO, L. X. SANTOS, W. O. PARRÉ, J. L. (2012). Uma Análise da Convergência Espacial do PIB per capita para os Municípios da Região Sul do Brasil (1999-2008). Anais: XV Encontro de Economia da Região Sul ANPEC SUL. Porto Alegre: Anpec.

SANTOS, G. C.(2010) Tratamento e extração dos microdados da PNAD. Notas de aula.

Disponível

em: http://www.academia.edu/1740581/Aula_de_Microdados_no_Stata

SILVA, P. L. do N., PESSOA, D. G. C. and LILA, M. F. (2002). Análise estatística de dados da PNAD: incorporando a estrutura do plano amostral. Ciência Saúde Coletiva, vol.7, no.4, p.659-670. ISSN 1413-8123.

SKINNER, C., Holt, D. and Smith, T. 1989. Analysis of Complex Surveys. John Wiley \& Sons.

SOLOW, R. M.(1956) A Contribution to the Theory of Economic Growth. Quaterly Journal of Economics, v. 70, n. 1, p. 65-94. 